\title{
Mapping visuospatial and self-motion perception functions in the left parietal lobe
}

\author{
Carlos Velásquez, MD, ${ }^{1}$ Elsa Goméz, $\mathrm{ST},{ }^{2}$ and Juan Martino, MD, PhD ${ }^{1}$ \\ Departments of ${ }^{1}$ Neurological Surgery and ${ }^{2}$ Psychiatry, Hospital Universitario Marqués de Valdecilla and \\ Fundación Instituto de Investigación Valdecilla, Santander, Spain
}

Parietal lobe functions include somesthesia, language, calculation, self-motion perception, and visuospatial awareness. In this video, the authors show the intraoperative mapping of a left parietal lobe for a low-grade glioma resection. Standard sensory and language mapping were performed. Interestingly, by using the "Line Bisection" task, subcortical stimulation of the gyrus angularis was repeatedly associated with ipsilateral spatial neglect, often described in the right parietal lobe. In a similar way, subcortical stimulation in a more posterior point elicited episodes of vertigo, probably due to stimulation of the superior longitudinal fasciculus. Both findings were useful to define the functional limit of the resection.

The video can be found here: https://youtu.be/ggGDRW_6u0A.

KEYWORDS visuospatial perception; intraoperative electrical stimulation; awake craniotomy; parietal lobe; glioma; video 